\title{
Massive hemoptysis managed by prolonged anticoagulation-free veno-venous extracorporeal membrane oxygenation with which thoracic surgeries were accompanied: a case report
}

Tomonori Kimura*, Tomoe Kobayashi, Fumimasa Kobori, Maria Goto, Mikako Takemitsu, Atsuko Tanaka, Jiro Sato and Shinji Isomine

\begin{abstract}
Background: Massive hemoptysis causing inadequate ventilation results in life-threatening consequences. We present a patient who developed respiratory insufficiency produced by bronchiectatic massive hemoptysis and underwent prolonged anticoagulation-free veno-venous extracorporeal membrane oxygenation ( $\mathrm{V}$-ECMO) during which thoracic surgeries were performed.

Case presentation: A 79-year-old woman suffered massive hemoptysis resulting in respiratory failure during fiberoptic bronchoscopy. Bronchial intubation followed by one lung ventilation failed to ensure adequate oxygenation. Anticoagulation-free W-ECMO, therefore, was installed immediately. Since conservative hemostatic measures including bronchial arterial embolization were not effective, resection of the culprit lung was performed while on VV-ECMO. Next day an exploratory thoracotomy and intercostal artery embolization were needed for recurrent bleeding. The W-ECMO was withdrawn after five days of operation.

Conclusions: Massive hemoptysis can be fatal and needs instantaneous and intensive treatments. In our case, longterm anticoagulation-free W-ECMO during which thoracic surgeries and endovascular interventions were performed provided a favorable outcome.
\end{abstract}

Keywords: Massive hemoptysis, Respiratory failure, Anticoagulation-free veno-venous extracorporeal membrane oxygenation, Thoracic surgeries, Endovascular interventions

\section{Background}

Massive hemoptysis is a rare but serious complication of bronchiectasis. As long as pulmonary bleeding remains uncontrolled, it causes deprivation of gas exchange area resulting in lethal outcomes [1, 2]. Insufficient

\footnotetext{
*Correspondence: mukira1115@gmail.com
}

Department of Anesthesiology and Intensive Care Medicine, Funabashi Municipal Medical Center, 1-21-1 Kanasugi, Funabashi, Chiba 273-8588, Japan oxygenation through native lungs would claim an installation of artificial lung support [3]. In addition, unless conservative hemostatic measures work such as bronchial tamponade and bronchial arterial embolization (BAE), surgical options including resection of the culprit lesion should be considered [1, 2]. We report a patient who developed respiratory failure due to massive hemoptysis and was successfully treated by prolonged anticoagulation-free veno-venous extracorporeal membrane oxygenation (VV-ECMO) in conjunction with 
both conservative and surgical hemostatic measures. The report is, to our knowledge, the first to describe the anesthetic and perioperative managements of a patient who suffered persistent hemoptysis-induced respiratory insufficiency surmounted by prolonged anticoagulation-free VV-ECMO.

\section{Case presentation}

A 79-year-old woman presented with recurrent cough and hemoptysis. A computed tomography (CT) and laboratory studies indicated bronchiectasis in the lower lobe of the left lung associated with allergic bronchopulmonary mycosis. One month later, a sudden and massive hemoptysis prompted an emergency fiberoptic bronchoscopy (FOB), which exhibited substantial bleeding in the left lung and its aspiration into the right lung. A single-lumen endotracheal tube was immediately placed in the right main bronchus to isolate the right lung, followed by mechanical one lung ventilation (OLV). We had to choose a single-lumen tube in the endoscopy room unequipped for emergency airway management. An emergency computed-tomography angiography (CTA) revealed a bulged left bronchial artery, urging BAE. The common trunk of bronchial arteries arose from the thoracic aorta (Fig. 1), complicating selective advancement of an embolization catheter. The BAE failed to achieve satisfactory hemostasis. During BAE, oxygenation worsened down to $\mathrm{SpO}_{2} 40 \%$. A shift to bilateral mechanical ventilation provided a slight amelioration in blood gas analysis at $\mathrm{F}_{\mathrm{i}} \mathrm{O}_{2}$ 0.35; $\mathrm{pH}$ 7.37, $\mathrm{PaCO}_{2}, 35.5 \mathrm{mmHg}, \mathrm{PaO}_{2}$

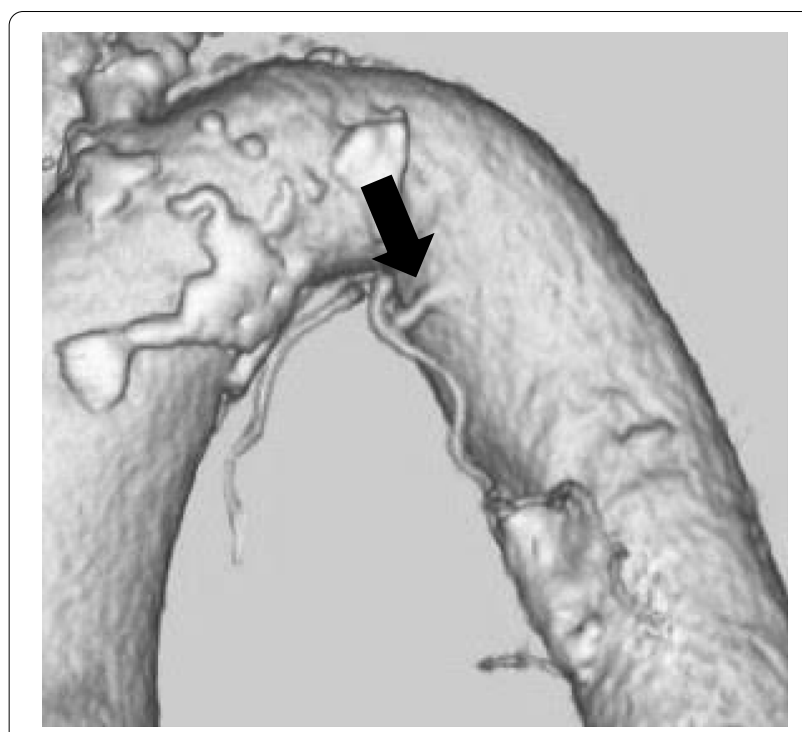

Fig. 1 Computed tomography angiography of bronchial arteries. The arrow indicates the common trunk of bronchial arteries which arose from the thoracic aorta
$104 \mathrm{mmHg}, \mathrm{HCO}_{3}{ }^{-} 20.2 \mathrm{mmol} / \mathrm{l}$, and $\mathrm{BE}-4.5 \mathrm{mmol} / \mathrm{l}$. However, we were afraid that severely decreased lung compliance produced by persistent blood afflux in both lungs would hamper sufficient and protective mechanical ventilation. We, thereby, decided to install VV-ECMO using a poly-2-methoxyethylacrylate (PMEA)-coated circuit (Capiox ${ }^{\circledR}$, TERUMO, Japan) withholding the use of anticoagulants with the setting of pump speed $1500 \mathrm{rpm}$, pump flow $2 \mathrm{~L} / \mathrm{min}, \mathrm{O}_{2}$ flow, $2 \mathrm{~L} / \mathrm{min}$. The coagulation system examinations following the installation of ECMO were activated partial thromboplastin time (APTT) $40 \mathrm{~s}$ and serum fibrinogen $158 \mathrm{mg} / \mathrm{dl}$.

Following 2 days, no apparent active bleeding observed let us confine only to performing FOBs for bronchial cleaning, hoping for spontaneous hemostasis. The finding and lowering extracorporeal membrane oxygenation (ECMO) support $\left(\mathrm{F}_{\mathrm{i}} \mathrm{O}_{2} 1.0\right.$ to 0.5$)$ suggested possible withdrawal from VV-ECMO despite chest $\mathrm{X}$-rays manifesting atelectasis in the whole left lung (Fig. 2A). On day 3 , however, an FOB found active rebleeding in the lateral and posterior basal bronchi, where thrombin solution was instilled. The single-lumen tube was replaced by a 35-Fr left-sided double-lumen endobronchial tube through which only the right lung was ventilated and the left lung was kept pressurized at a constant airway pressure $10 \mathrm{cmH}_{2} \mathrm{O}$ with $100 \% \mathrm{O}_{2}$, intending astriction. Notwithstanding the efforts, we thought such conservative means were only palliative and a radical surgical measure should be adopted. In the meantime, ECMO weaning trials were carried out in accordance with the Extracorporeal Life Support Organization guideline [4], indicating possible weaning. The ECMO was, however, kept operated at the minimal setting, pump speed 1250 $\mathrm{rpm}$, pump flow $1.5 \mathrm{~L} / \mathrm{min}, \mathrm{O}_{2}$ flow $0.5 \mathrm{~L} / \mathrm{min}$, in preparation for surgery-associated worsening of gas exchange and unexpected hemorrhage. Preoperative total amounts of blood products transfused were fresh frozen plasma (FFP) 6 units, packed red cells (PRC) 6 units and platelets 10 units. The preoperative APTT was $42 \mathrm{~s}$ and serum fibrinogen $173 \mathrm{mg} / \mathrm{dl}$.

On day 4, resection of the left lower lung lobe was scheduled under inhalational anesthesia with sevoflurane while the patient was on ECMO. We were concerned about unstable depth of intravenous anesthesia produced by abrupt changes in hemodynamics and circulation volume. Depth of anesthesia was closely monitored with the bispectral index $\left(\mathrm{BIS}^{\circledR}\right.$, Medtronic, USA). The VV-ECMO remained well-controlled during the surgery without major cardiovascular or respiratory events. The surgery achieved considerable hemostasis, with the operation duration $3 \mathrm{~h} 51 \mathrm{~min}$ and intraoperative bleeding volume $863 \mathrm{ml}$. She was transfused with FFP 8 units, PRC 10 units, and platelets 20 units. Postoperative chest 

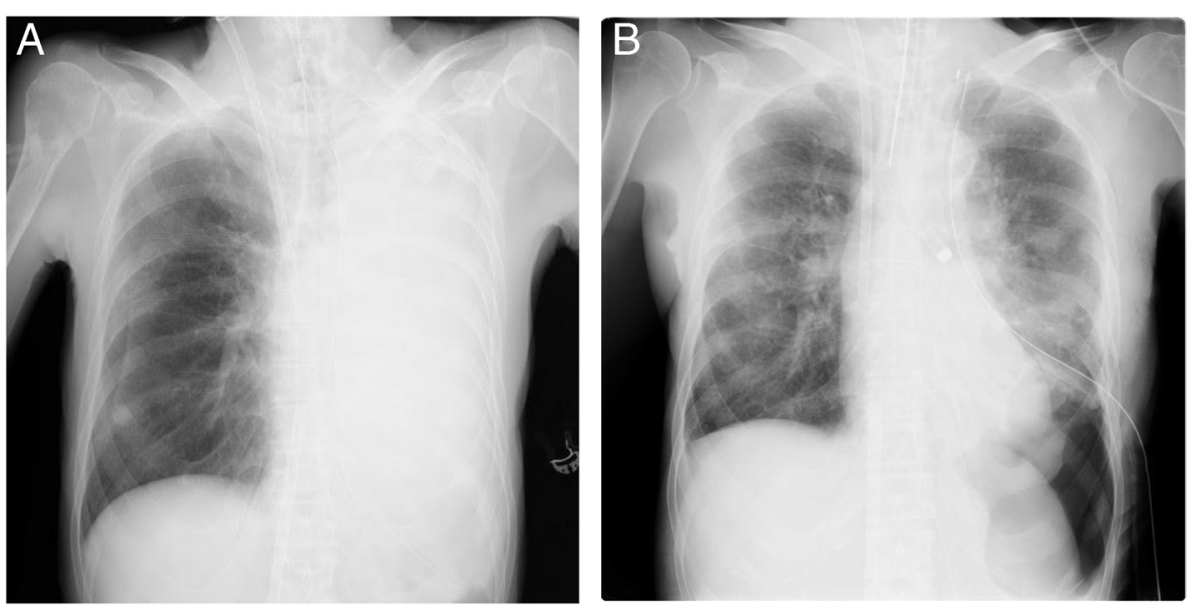

Fig. 2 A Chest X-ray taken on day 2. It showed diffuse consolidation of the left lung due to massive hemoptysis, indicating whole lung atelectasis. B Chest X-ray taken after surgery on day 4 . It showed good aeration in the resting left upper lung

X-ray showed good aeration in the resting left upper lung (Fig. 2B). Bilateral mechanical ventilation presented a marked improvement in gas exchange. However, VVECMO still remained operated in the postoperative ICU at the minimal setting since unstable hemodynamics and slowly progressing anemia were sustained.

On day 5, the patient developed a hematoma in the left thoracic wall. An exploratory thoracotomy was performed, achieving hemostasis. Intraoperative bleeding of $2500 \mathrm{ml}$ was compensated by transfusions of FFP 18 units, PRC 12 units and platelets 20 units. Serum fibrinogen was below $100 \mathrm{mg} / \mathrm{dl}$ preoperatively but recovered to $132 \mathrm{mg} / \mathrm{dl}$ after surgery. For inspection of intravascular emboli formed possibly after prolonged anticoagulation-free ECMO, a postoperative CTA was performed and found, instead, extravasation of contrast medium from intercostal arteries. Transcatheter arterial embolization (TAE) provided a dramatic hemodynamic stability, enabling weaning from VV-ECMO on the same day. Eventually, VV-ECMO was kept operated without anticoagulation for as long as 5 days. On day 6 , a CTA detected floating thrombi in the inferior vena cava and bilateral popliteal veins, which required a continuous heparin administration. She was extubated on day 8 and transferred to a general ward on day 9. She was discharge uneventfully from the hospital on day 53 .

\section{Discussion}

There are case studies on patients who underwent pulmonary surgery while on VV-ECMO for major airway involvements [5-7]. Preoperative anticipation of the need of temporary interruptions of lung ventilation and gas exchange failure due to lung comorbidities were main reasons for elective and prophylactic use of intraoperative
VV-ECMO. In our patient, on the contrary, ECMO was operated continuously beforehand, started at the instance of massive lung bleeding, and carried out even during and after two surgeries.

We needed to balance among prevention of impermissible amounts of bleeding, safe operation of ECMO and assurance of gas exchange via both native lung ventilation and ECMO. We chose anticoagulation-free VV-ECMO, afraid of anticoagulant-induced exacerbation of bleeding.

Albeit a variety of ECMO surface materials circumventing the use of systemic anticoagulants have long been under development [8], no stable techniques have yet been established for long-term anticoagulation-free operation of VV-ECMO. Although the PMEA-coated system we employed is indicated to reduce circuit-related adverse effects such as inflammatory responses and thrombo-coagulopathies, these findings were obtained only by either in vitro studies or short-term uses. In addition, only a few case-based studies reported feasibility of long-term VV-ECMO with low-dose anticoagulant uses $[9,10]$. Therefore, we needed to pay considerable attention to coagulation status in the circulation. We monitored blood coagulability solely by serum fibrinogen and APTT accompanied by a careful observation of the status of blood stream in ECMO circuit. After all, fortunately we did not need to exchange the anticoagulation-free circuit for as long as five days. It is, however, undeniable that indiscernible changes occurred in the coagulationfibrinolysis system which would lead to clotting in the circuit, oxygenator failure, vessel embolism, and hemorrhagic events, as indicated by deep venous thrombosis detected on day 6 .

Massive hemoptysis is known to present higher mortality rates with greater amounts of bleeding, reported to be 
as high as 78\% [1]. We, therefore, kept ECMO operated at the minimal setting even when considered unnecessary to secure a transfusion route before bleeding is definitely controlled. Prolonged VV-ECMO in hemorrhagic patients would be a challenge in balancing between thrombotic and bleeding events. Although we adopted an anticoagulation-free operation of ECMO in an attempt to avoid bleeding risk, it would increase the risk of lifethreatening thrombo-coagulopathies such as pulmonary artery thromboembolism. Therefore, other choices which have a potential to circumvent both risks concurrently would have to be considered such as intermittent operation of ECMO and use of direct thrombin inhibitors including argatroban [11].

There are considerations regarding the choice of anesthesia while running ECMO. It may be subject to pharmacokinetic instabilities in intravenous anesthetics produced by changes in circulating blood volume due to intraoperative bleeding and extracorporeal circulation, and drug sequestration by ECMO circuit [12]. We, therefore, employed inhalational anesthesia. However, we might need to keep in mind the possibility of unstable delivery of volatile anesthetics in patients with severely reduced pulmonary area for gas exchange as in this case.

The BAE has been considered a first-line therapy for hemoptysis. It is, however, known to be not necessarily successful because of radiographic visualization of noncommunicating arteries, perfusion from collaterals, aberrant bronchial arteries, etc. Moreover, embolization of bronchial to spinal artery collaterals would mislead to a serious consequence $[1,2]$. We, therefore, decided not to reinstitute BAE after the failure of the first attempt.

In conclusion, considerable experience and expertise in the management of VV-ECMO would enable safe perioperative management of patients with massive pulmonary bleeding resulting in otherwise non-survivable respiratory failure.

\begin{abstract}
Abbreviations
BAE: Bronchial arterial embolization; WV-ECMO: Veno-venous extracorporeal membrane oxygenation; CT: Computed tomography; FOB: Fiberoptic bronchoscopy; OLV: One lung ventilation; CTA: Computed-tomography angiography; PMEA: Poly-2-methoxyethylacrylate; APTT: Activated partial thromboplastin time; ECMO: Extracorporeal membrane oxygenation; FFP: Fresh frozen plasma; PRC: Packed red cells; TAE: Transcatheter arterial embolization.
\end{abstract}

\section{Acknowledgements}

Not applicable.

\section{Authors' contributions}

TK was the main attending anesthesiologist of the patient and wrote the manuscript. MG, TK, and MK conducted anesthesia and perioperative care with TK and reviewed the manuscript. AT and MT advised on anesthesia and reviewed the manuscript. JS assisted manuscript writing and edited the manuscript. SI, director of the department, advised on treatments and reviewed the manuscript. All authors read and approved the final manuscript.
Funding

Nothing to declare.

Availability of data and materials

Not applicable.

\section{Declarations}

Ethics approval and consent to participate

Not applicable.

\section{Consent for publication}

Written informed consent was obtained from the patient for publication of this case report and accompanying images.

\section{Competing interests}

The authors declare that they have no competing interests.

Received: 1 December 2021 Revised: 9 February 2022 Accepted: 11 February 2022

Published online: 21 February 2022

References

1. Sakr L, Dutau H. Massive hemoptysis: an update on the role of bronchoscopy in diagnosis and management. Respiration. 2010;80:38-58.

2. Jin F, Li Q, Bai C, Wang H, Li S, Song Y, et al. Chinese expert recommendation for diagnosis and treatment of massive hemoptysis. Respiration. 2020;99:83-92.

3. King CS, Whitney Brown A, Aryal S, Ahmad K, Donaldson S. Critical care of the adult patient with cystic fibrosis. Chest. 2019;155:202-14.

4. Tonna JE, Abrams D, Brodie D, Greenwood JC, Rubio Mateo-Sidron JA, Usman A, et al. Management of adult patients supported with venovenous extracorporeal membrane oxygenation (VV ECMO): Guideline from the Extracorporeal Life Support Organization (ELSO). ASAIO J. 2021;67:601-10.

5. Miyamoto Y, Nagamine Y, Goto T, Kurahashi K. Intraoperative venovenous extracorporeal membrane oxygenation during thoracic surgery that requires 1-lung ventilation: a case report. A\&A Practice. 2018;10:79-82.

6. Suk P, Sramek V, Cundrle I Jr. Extracorporeal membrane oxygenation use in thoracic surgery. Membranes. 2021;11:416-24.

7. Foong TW, Ramanathan K, Man Chan KK, MacLaren G. Extracorporeal membrane oxygenation during adult noncardiac surgery and perioperative emergencies: a narrative review. J Cardiothorac Vasc Anesth. 2021;35:281-97.

8. Willers A, Arens J, Mariani S, Pels H, Maessen JG, Hackeng TM, et al. New trends, advantages and disadvantages in anticoagulation and coating methods used in extracorporeal life support devices. Membranes. 2021;11:617-31.

9. Seeliger B, Dobler M, Friedrich R, Stahl K, Kuhn C, Bauersachs J, et al. Comparison of anticoagulation strategies for veno-venous ECMO support in acute respiratory failure. Crit Care. 2020;24:701-11.

10. Kurihara C, Walter JM, Karim A, Thakkar S, Saine M, Odell DD, et al. Feasibility of venovenous extracorporeal membrane oxygenation without systemic anticoagulation. Ann Thorac Surg. 2020;110:1209-15.

11. Kato C, Oakes M, Kim M, Desai A, Olson SR, Raghunathan V, et al. Anticoagulation strategies in extracorporeal circulatory devices in adult populations. Eur J Haematol. 2021;106:19-31.

12. Fierro MA, Daneshmand MA, Bartz RR. Perioperative management of the adult patient on venovenous extracorporeal membrane oxygenation requiring noncardiac surgery. Anesthesiology. 2018;128:181-201.

\section{Publisher's Note}

Springer Nature remains neutral with regard to jurisdictional claims in published maps and institutional affiliations. 